\title{
R3. OBTAINING A RECOMBINANT ANTIGEN OF Taenia crassiceps FOR USE IN IMMUNODIAGNOSIS.
}

Regina Helena Saramago Peralta ${ }^{1}$; Thiago Rodrigues Lima ${ }^{1}$; Ronaldo Mohana -Borges²; José Mauro Peralta².

${ }^{1}$ Universidade Federal Fluminense;

${ }^{2}$ Universidade Federal do Rio de Janeiro.

INTRODUCTION Neurocysticercosis (NC) is caused by the presence of Taenia solium in the CNS. Diagnosis requires proper evaluation of clinical, neuroimaging and serological data, in the correct epidemiological context. The development of immunological assays with purified and recombinant proteins and synthetic peptides can improve the assays efficiency, increasing sensitivity and specificity. Studies of crossreactivity between Taenia crassiceps and T. solium antigens confirmed that both share common epitopes.

OBJECTIVE The aim of this study was to establish protocols for cloning, expression and purification of the gp14 recombinant protein of T. crassiceps and evaluate its diagnosis potential.

METHODOLOGY The cloning of gp14 DNA was performed through synthetic DNA technology. The DNA was inserted into the vector pET21dEZ and transformed into the Escherichia coli strain DH5a. The plasmid was inserted into competent strains of $E$. coli. The recombinant $14 \mathrm{kDa}$ protein purity was assessed by SDS-PAGE and also by Western Blot. The cloning was confirmed by sequencing. The SDS-PAGE and Western Blot analysis showed a band corresponding to the gp14r. The cloning using synthetic DNA technology allowed us to obtain recombinant protein in sufficient amount to use in immunoassays.

RESULTS The gp14r was used in ELISA and LIApep for detection of antibodies of anti-T. solium in 8 samples of NC patients, with active disease; 25 samples of patients with others parasites, including hydatid disease; 8 samples of healthy individuals and 21 samples of pigs suspected of $\mathrm{NC}$. All samples of control groups were no reactive in the tests and the NC patients were reactive.

CONCLUSION The results showed a good performance and consist in an important tool for the NCC diagnosis.

KEYWORDS neurocysticercosis, immunoassays, recombinant proteins. 